\title{
Aumento da Eficiência Espectral de Sistemas com Codificação Wavelet
}

\author{
Eline A. Santos, Edmar C. Gurjão e Francisco M. de Assis
}

\begin{abstract}
Resumo- $O$ aproveitamento da ortogonalidade entre linhas de uma matriz wavelet tem sido explorada para obter sistemas de comunicação sem fio que apresentam desempenho comparável, e em alguns casos superior, aos que utilizam códigos espáciotemporais. Entretanto, uma limitação dos sistemas wavelet propostos até o momento é a eficiência espectral (EE) que fica limitada a $1 \mathrm{bit} / \mathrm{s} / \mathrm{Hz}$. A combinação de símbolos wavelet em um símbolo do canal pode aumentar a eficiência espectral desses sistemas, e neste trabalho propomos o uso da função geradora de momentos para, a partir de uma matriz de codificação, obter as sequências de símbolos wavelet e suas probabilidades conjuntas, e através de um exemplo, mostra-se que a combinação de pares de símbolos wavelet permite aumentar a EE para 2 bits/s/Hz. O método proposto é geral e permite determinar, para uma dada matriz de codificação wavelet quais sequências de tamanho $N$ podem ser obtidas, o que abre a possibilidade de realizar combinações de sequência de símbolos wavelet para obter sistemas com eficiência espectral maiores.
\end{abstract}

Palavras-Chave-Wavelet, codificação, eficiência espectral, função geradora, momentos.

Abstract-The row orthogonality of wavelet matrix has been used to obtain wireless communication systems with performance comparable, and in some cases superior, to space-time encoded systems. In spite of their good performances, the spectral efficiency (SE) limited to $1 \mathrm{bits} / \mathrm{s} / \mathrm{HZ}$ is them main drawback of these systems. The combination of sequences of wavelet symbols transmitted in one channel symbols may increase the SE, and in this work we apply the moment generating function to obtain, for a given wavelet matrix, the sequence of wavelet symbols and their joint probabilities. As an example we combine two consecutive wavelet symbols and we obtain a $2 \mathrm{bits} / \mathrm{s} / \mathrm{Hz}$ system that maintains the good performance of wavelet systems. The proposed method is general and it permits to obtain the sequences of length $N$ and their joint probability generated by using a given wavelet matrix, and it opens the possibility to project new sequence combinations to obtain systems with high SE.

Keywords-Wavelet, encoding, spectral efficiency, moment generating function.

\section{INTRODUÇÃO}

A utilização das propriedades de ortogonalidade entre as linhas das matrizes wavelet para transmissão digital em canais com desvanecimento foi proposta em 1992 por Tzannes et al [1]. Duas vantagens principais podem ser identificadas neste tipo de codificação. A primeira é diversidade temporal que a técnica proporciona, posto que a informação contida em cada símbolo da fonte é mapeado "naturalmente" em uma longa sequência de símbolos codificados, ao mesmo tempo

Eline A. Santos, Doutoranda no Programa de Pós-Graduação em Engenharia Elétrica, PPgEE/UFCG, Edmar C. Gurjão e Francisco M. de Assis, Departamento de Engenharia Elétrica, Universidade Federal de Campina Grande, Paraíba, Brasil. E-mails: eline.santos@ee.ufcg.edu.br, ecandeia@dee.ufcg.edu.br, fmarcos@dee.ufcg.edu.br. a demodulação é feita com uso de um bloco simples de filtros casados. A segunda vantagem é a facilidade para implementar a codificação com taxas variáveis, por intermédio de uma simples alteração na "superposição" das linhas da matriz de codificação [2].

Entretanto a codificação por matrizes wavelet apresenta algumas dificuldades. Por exemplo, a probabilidade dos símbolos codificados não é uniforme e a cardinalidade do conjunto de símbolos a serem transmitidos aumenta com o número de colunas da matriz wavelet usada na codificação. Sendo que o número de colunas determina a quantidade de símbolos codificados influenciados por um mesmo símbolo da fonte, logo o ganho de desempenho da codificação wavelet depende fortemente deste fator. Estas características vão requerer maiores cuidados na construção de constelações de sinais convenientes para a transmissão. Diversas pesquisas foram realizadas neste tema com resultados apresentados na literatura especializada [3], [5], [4].

Uma outra dificuldade encontrada para a popularização do uso deste tipo de codificação é a eficiência espectral limitada superiormente em $1 \mathrm{bit} / \mathrm{s} / \mathrm{Hz}$ para a maioria dos esquemas propostos, e.g, [7]. Um dos obstáculos é que a associação ingênua de múltiplos bits da fonte para uma posterior codificação por matrizes wavelet conduz a uma proliferação de sinais necessários para a transmissão bem como a redução da distância euclidiana entre eles, o que afeta diretamente o desempenho.

Como cada símbolo wavelet transporta em média 1 bit de informação, a abordagem considerada neste trabalho é a combinação de blocos desses símbolos para gerar uma nova representação que transporte em média mais bits. Para aplicar essa abordagem, o primeiro passo é determinar quais os blocos de símbolos podem ser gerados e quais são suas probabilidades conjuntas. Para determinar os blocos mencionados e suas probabilidades introduzimos uma função geradora de momentos ligeiramente modificada, que pode ser usada para blocos de símbolos de tamanho arbitrário. Exemplificamos o uso desta função para blocos de dois símbolos consecutivos em um sistema com eficiência espectral de 2 bits/s/Hz.

Da junção de dois símbolos consecutivos, observou-se que a constelação dos sinais é obtida simplesmente considerando os pares de símbolos codificados como componentes em fase e em quadradura, com a conveniente normalização da energia média. As propriedades "geométricas" da constelação resolvem um problema encontrado em formulações anteriores, e.g., [5], qual seja, a ocorrências de erros de grande magnitude. A solução apresentada assemelha-se aos esquemas OFDM no sentido em que a saída da fonte depois de passar por uma 
transformação é mapeada diretamente no espaço dos sinais. No caso do OFDM a transformada discreta inversa de Fourier é utilizada [6], no sistema aqui apresentado a transformada é materializada pela codificação wavelet.

A distribuição dos pares de símbolos (e portanto a constelação de sinais) é obtida por intermédio de uma função geradora ordinária cujas configurações combinatórias são representadas por séries formais da sequência de símbolos codificados [8, p. 29ss]. A função geradora proposta pode ser usada para encontrar a distribuição conjunta de $N$-úplas quaisquer com $N \geq 2$. Entretanto suas propriedades não foram exploradas para além do uso para obter as distribuições conjuntas, em particular não foi explorado seu uso para o cálculo de momentos.

Na Seção II a codificação wavelet é apresentada, na Seção III a função função geradora é definida e utilizada para obter a distribuição de probabilidades de pares de símbolos codificados. Na seção IV mostra-se a obtenção de um sistema com eficiência $2 \mathrm{bits} / \mathrm{s} / \mathrm{Hz}$. Os resultados obtidos são apresentadas na Seção V e as conclusões e perspectivas para desdobramentos deste trabalho são apresentados na Seção VI.

\section{CODIFICAÇÃo WAVELET}

Uma matriz de codificação wavelet (MCW) de ordem $m$ e gênero $g$ é definida como

$$
\mathbf{A}=\left(\begin{array}{ccc}
a_{0}^{0}, & \cdots, & a_{m g-1}^{0} \\
a_{0}^{1}, & \cdots, & a_{m g-1}^{1} \\
\vdots & \ldots & \vdots \\
a_{0}^{m-1} & \cdots, & a_{m g-1}^{m-1}
\end{array}\right)
$$

Neste trabalho utilizamos uma MCW cujos coeficientes $\left(a_{k}^{j}\right) \in\{+1,-1\}$, e cujas linhas satisfazem a condição de escalonamento modificada [1]

$$
\sum_{k=0}^{m g-1} a_{k}^{j}=m \sqrt{g} \delta_{0, j}, \quad 0 \leq j \leq m-1
$$

$$
\begin{array}{r}
\sum_{k=0}^{m g-1} a_{[k+m l]}^{j} a_{\left[k+m l^{\prime}\right]}^{j^{\prime}}=m g \delta_{j, j^{\prime}} \delta_{l, l^{\prime}}, \\
0 \leq j, j^{\prime} \leq m-1 \\
0 \leq l, l^{\prime} \leq m-1
\end{array}
$$

sendo $\delta_{j, j^{\prime}}$ o delta de Kronecker e a notação $[k+m l]$ significa $(k+m l) \bmod m g$

Equação (3) indica que as linhas da MCW de ordem $m$ são mutualmente ortogonais. Ela também indica que a ortogonalidade se mantém caso as linhas sejam deslocadas de $l m$ posições sendo $0<l \leq g-1$. Essas ortogonalidades são as condições básicas para a codificação de canal wavelet.

Consideraremos os bits de informação $x_{n} \in\{+1,-1\}$, e uma MCW $A=\left(a_{k}^{j}\right)$ de ordem $m$ e gênero $g$. No processo de codificação wavelet, cada um dos bits de um conjunto de $m$ bits é multiplicado por linhas distintas da MCW, e os resultados dessas multiplicações são deslocados de $m$ posições e adicionados para gerar os símbolos wavelet ${ }^{1}$. O procedimento de codificação é ilustrado na Tabela I para uma MCW de ordem 2 e gênero 4.

\footnotetext{
${ }^{1} \mathrm{O}$ codificador wavelet poder ser representado como um banco de filtros, como mostrado em [5]
}

TABELA I

EXEMPLO DE CODIFICAÇÃO DE CANAL WAVELET

\begin{tabular}{|c|c|c|c|c|c|c|c|c|}
\hline 1 & 2 & 3 & 4 & $\cdots$ & 8 & 9 & 10 & $\cdots$ \\
\hline$x_{1} a_{0}^{0}$ & $x_{1} a_{1}^{0}$ & $x_{1} a_{2}^{0}$ & $x_{1} a_{3}^{0}$ & $\cdots$ & $x_{1} a_{7}^{0}$ & & & \\
$x_{2} a_{0}^{1}$ & $x_{2} a_{1}^{1}$ & $x_{2} a_{2}^{1}$ & $x_{2} a_{3}^{1}$ & $\cdots$ & $x_{2} a_{7}^{1}$ & & & \\
& & $x_{3} a_{0}^{0}$ & $x_{3} a_{1}^{0}$ & $\cdots$ & $x_{3} a_{5}^{0}$ & $x_{3} a_{6}^{0}$ & $x_{3} a_{7}^{0}$ & \\
& & $x_{4} a_{0}^{1}$ & $x_{4} a_{1}^{1}$ & $\cdots$ & $x_{4} a_{5}^{1}$ & $x_{4} a_{6}^{1}$ & $x_{4} a_{7}^{1}$ & \\
& & & & & $\vdots$ & $\vdots$ & $\vdots$ & $\cdots$ \\
\hline$y_{1}$ & $y_{2}$ & $y_{3}$ & $y_{4}$ & $\cdots$ & $y_{8}$ & $y_{9}$ & $y_{10}$ & $\cdots$ \\
\hline
\end{tabular}

O símbolo wavelet produzido no instante $n$ é igual a soma da $n$-ésima coluna das mensagens codificadas, mostradas na Tabela I, e sendo uma soma de inteiros \pm 1 [5] tem valores no conjunto $\{-m g,-m g+2, \ldots,-m g+2 k, \ldots, 0, \ldots, m g-$ $2, m g\}$. Observe que a informação de um bit é espalhada em vários símbolos wavelet, e com isso consegue-se uma memória que ajuda a proteger dos efeitos do canal.

No processo de codificação, $m$ bits de informação são codificados em $m$ símbolos wavelet e são transmitidos em $m$ intervalos de sinalização, então a eficiência espectral do sistema é de $1 \mathrm{bit} / \mathrm{s} / \mathrm{Hz}$.

Os bits de informação $\left\{x_{n}\right\}$ podem ser recuperados da sequência de símbolos recebidos usando um banco de $m$ correlatores de comprimento $m g$ casados com as $m$ linhas da MCW, como mostrado em [5], e a simplicidade da decodificação por correlação é uma das principais vantagens da técnica de codificação wavelet [1].

\section{USO DA FUNÇÃO GERADORA DE MOMENTOS PARA OBTER SEQUÊNCIAS DE SÍMBOLOS WAVELET}

Apesar da facilidade de codificação e decodificação, aliado ao bom desempenho em canais com desvanecimento, a codificação wavelet tem sido criticada pela eficiência espectral, $1 \mathrm{bit} / \mathrm{s} / \mathrm{Hz}$, que é fixa e baixa quando comparada a sistemas de transmissão sem fio com desempenhos similares.

Na busca por técnicas para melhorar a eficiência espectral, partiu-se do pressuposto básico de associar mais de um bit de informação a um símbolo do canal, e isto pode ser feito pela combinação de bits antes da codificação wavelet. Entretanto, observando que em cada passo do processo de codificação $m$ símbolos wavelet são produzidos, e que cada símbolo transporta em média 1 bit de informação, se combinarmos os símbolos wavelet em um único símbolo do canal, cada novo simbolo do canal produzido irá transportar mais de um bit de informação, aumentando a eficiência espectral do sistema, essa é a técnica usada nesse trabalho.

Para que seja possível analisar o desempenho do sistema que transmite símbolos wavelet gerados em sequência e agrupados em um símbolo do canal, vamos determinar quais sequências de símbolos são possíveis, e qual a distribuição de probabilidade conjunta desses agrupamentos, o que é feito a seguir.

\section{A. Distribuição conjunta dos agrupamentos de símbolos Wavelet}

Para usar a função geradora dos momentos e obter a distribuição de probabilidade dos agrupamentos de símbolos 
wavelet consecutivos, vamos considerar uma sequência de símbolos wavelet que inicia no instante $n$, e tem comprimento $N$ representada por $y_{n}, y_{n+1}, y_{n+2}, \ldots y_{n+N-1}$, então lembrando que [9]:

$$
G_{X}(z) \triangleq \mathbb{E} z^{X}
$$

Definindo a série formal em $D$ com variáveis aleatórias $\left(y_{n}, y_{n+1}, y_{n+2}, \ldots y_{n+N-1}\right)$ :

$$
Y(D)=y_{n}+y_{n+1} D+y_{n+2} D^{2}+\ldots+y_{n+N-1} D^{N-1}
$$

em que $D$ marca a posição de um símbolo em uma sequência, e aplicando uma generalização da função geratriz onde a variável aleatória $X$ é trocada por $Y(D)$, temos:

$$
G_{Y(D)}(z) \triangleq \mathbb{E} z^{Y(D)} .
$$

Expandindo esta função temos a sequência de comprimento $N$ de possíveis símbolos wavelet e a distribuição conjunta dessas sequências.

Como exemplo vamos determinar a distribuição conjunta de dois símbolos $(N=2)$ consecutivos para uma MCW $2 \times 8$. Neste caso, temos dois regimes de codificação, o instante $n$ é associado como regime de codificação par, e o instante $n+1$ com o regime de codificação ímpar, dados por:

$$
\begin{aligned}
Y_{2 p}[n] & =a_{0}^{0} X_{n}+a_{2}^{0} X_{n-2}+a_{4}^{0} X_{n-4}+a_{6}^{0} X_{n-6}+a_{0}^{1} X_{n+1} \\
& +a_{2}^{1} X_{n-1}+a_{4}^{1} X_{n-3}+a_{6}^{1} X_{n-5}
\end{aligned}
$$$$
Y_{2 p+1}[n+1]=a_{1}^{0} X_{n}+a_{3}^{0} X_{n-2}+a_{5}^{0} X_{n-4}+a_{7}^{0} X_{n-6}
$$$$
+a_{1}^{1} X_{n+1}+a_{3}^{1} X_{n-1}+a_{5}^{1} X_{n-3}+a_{7}^{1} X_{n-5}
$$

Substituindo os valores da MCW temos:

$$
\begin{aligned}
Y_{2 p}[n]= & X_{n}+X_{n-2}+X_{n-4}-X_{n-6}+X_{n+1} \\
+ & X_{n-1}-X_{n-3}+X_{n-5} \\
Y_{2 p+1}[n+1] & =X_{n}-X_{n-2}+X_{n-4}+X_{n-6}+X_{n+1} \\
& -X_{n-1}-X_{n-3}-X_{n-5}
\end{aligned}
$$

Então, definido as variáveis

$$
Y(D)=Y_{2 p}[n]+Y_{2 p+1}[n+1] D
$$

a probabilidade do par de símbolos será encontrada usando a função geratriz dada por

$$
\begin{aligned}
G_{Y(D)}(z) & =\mathbb{E} z^{Y(D)} \\
& =\mathbb{E} z^{Y_{2 p}[n]+Y_{2 p+1}[n+1] D}
\end{aligned}
$$

substituindo $Y_{2 p}[n]$ e $Y_{2 p+1}[n+1]$ por suas expressões (7) e (8) respectivamente, e arrumando as expressões temos

$$
\begin{aligned}
G_{Y(D)}(z)= & \mathbb{E} z^{(1+D) X_{n+1}+(1+D) X_{n}+(1-D) X_{n-1}} \\
& +(1-D) X_{n-2}+(-1-D) X_{n-3}+(1+D) X_{n-4} \\
& +(1-D) X_{n-5}+(-1+D) X_{n-6}
\end{aligned}
$$

Considerando um fonte de bits $\left(X_{n}\right)$ i.i.d. e

$$
\begin{aligned}
G_{Y(D)}(z)= & G_{X_{n+1}}\left(z^{1+D}\right) G_{X_{n}}\left(z^{1+D}\right) \\
& \ldots G_{X_{n-5}}\left(z^{1-D}\right) G_{X_{n-6}}\left(z^{-1+D}\right)
\end{aligned}
$$

Para uma fonte binária $x_{i} \in \pm 1$ e $P\left[x_{i}=1\right]=P\left[x_{i}=\right.$ $-1]=1 / 2$, então:

$$
G_{X_{i}}\left(z^{f(D)}\right)=\frac{1}{2}\left(z^{f(D)}+z^{-f(D)}\right)
$$

Neste caso

$$
G_{Y(D)}(z)=\frac{1}{2^{8}}\left(z^{1-D}+z^{-1+D}\right)^{4}\left(z^{1+D}+z^{-1-D}\right)^{4}
$$

e expandindo essa equação em $z$ encontramos tanto os pares de símbolos wavelet que podem ser gerados em sequência, quanto a distribuição conjunta desse pares. O expoentes da variável $z$ representam os possíveis pares de símbolos, e os coeficientes do polinômio correspondem às probabilidade de acordo com o expoente em $z$. Para nosso exemplo encontramos

$$
\begin{aligned}
G_{Y(D)}(z) & =\frac{1}{2^{8}}\left(36 z^{0+0 D}+24 z^{2+2 D}+24 z^{2-2 D}\right. \\
& +24 z^{-2+2 D}+24 z^{-2-2 D}+16 z^{0+4 D} \\
& +16 z^{0-4 D}+16 z^{-4+0 D}+6 z^{4+4 D}+6 z^{4-4 D} \\
& +6 z^{-4+4 D}+6 z^{-4-4 D}+4 z^{2+6 D}+4 z^{6+2 D} \\
& +4 z^{2-6 D}++4 z^{-6+2 D}+4 z^{-2+6 D}+4 z^{6-2 D} \\
& +4 z^{-2-6 D}+4 z^{-6-2 D}+z^{0+8 D}+z^{8+0 D} \\
& \left.+z^{0-8 D}+z^{-8+0 D}\right) .
\end{aligned}
$$

A Equação (15) fornece os pares de símbolos como os expoentes da variável $z$, e respectivas probabilidades conjuntas como o coeficiente da variável correspondente. Os pares de símbolos e as respectivas probabilidades estão representados na Tabela II.

TABELA II

PARES DE SÍMBOLOS WAVELET E SUAS RESPECTIVAS PROBABILIDADES

\begin{tabular}{|c|l|}
\hline Pares de símbolos & $\begin{array}{l}\text { Probabilidade } \\
\left(\times 2^{-8}\right)\end{array}$ \\
\hline$(0,0)$ & 36 \\
\hline$(2,2),(2,-2),(-2,2),(-2,-2)$ & 24 \\
\hline$(0,4),(0,-4),(4,0),(-4,0)$ & 16 \\
\hline$(4,4),(4,-4),(-4,4),(-4,-4)$ & 6 \\
\hline$(2,6),(6,2),(2,-6),(-6,2),(-2,6),(6,-2),(-2,-6),(-6,-2)$ & 4 \\
\hline$(0,8),(8,0),(0,-8),(-8,0)$ & 1 \\
\hline
\end{tabular}

\section{AgRupamento de Símbolos WAVElet Para AUMENTAR A EFICIÊNCIA ESPECTRAL}

A Equação (15) fornece os possíveis pares de símbolos wavelet e suas probabilidades, listados na Tabela II, e se desenharmos esses pares de símbolos como pontos no plano $\mathbb{R}^{2}$ obtemos a estrutura representada na Figura 1, onde também estão indicadas as probabilidades conjuntas. Observa-se a regularidade da estrutura obtida, um quadrado, e que o seu formato se assemelha a uma constelação retangular porém rotacionada, de sinais para modulação. Dessa forma, ao invés de fazer um mapeamento das sequências de símbolos em pontos de uma constelação de sinais de uma modulação, decidiu-se utilizar a distribuição de pares obtidas obtida como a constelação de sinais a serem transmitidos pelo canal. 
O esquema que utiliza a constelação obtida pelo mapeamento dos agrupamentos de símbolos wavelet, como o exemplificado na Figura 1, faz o mapeamento da sequência de bits da fonte em símbolos do canal, e assim pode ser considerado uma codificação fonte-canal.

Além disso, a "constelação" proposta tem características interessantes, pois pontos de alta probabilidade correspondem a sinais de baixa energia, e pares de símbolos wavelet próximos são mapeados para pontos vizinhos na espaço de sinais do canal, e com isso os erros impostos pelo canal que causem trocas de símbolos wavelet, que causam grande influência no desempenho, são amenizados.

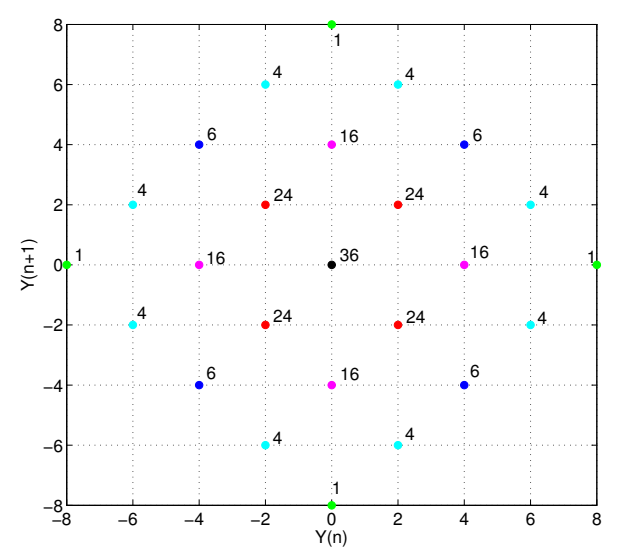

Fig. 1. Pares de Símbolos wavelet no Plano $\mathbb{R}^{2}$ para MCW $2 \times 8$. A probabilidade do par é igual ao rótulo numérico multiplicado por $2^{-8}$.

O desempenho da codificação conjunta em canais com desvanecimento será analisado na próxima seção.

\section{Resultados}

Seguindo o esquema de codificação conjunta fonte-canal proposto na seção anterior, vamos utilizar os pontos no plano $\mathbb{R}^{2}$ como uma constelação de sinais que serão transmitidos pelo canal.

Nas simulações consideramos uma fonte binária i.i.d.,canal com desvanecimento Rayleigh plano e lento e ruído aditivo Gaussiano branco (AWGN - do inglês Additive White Gaussian Noise). No receptor considera-se conhecimento perfeito do canal e usa-se o critério Maximum a posteriori - MAP.

Para obter os resultados vamos usar a constelação proposta na Figura 1, e outra obtida para uma matriz $2 \times 128$, e em ambos os casos cada dois símbolos wavelet serão associados a um simbolo do canal, e assim teremos sistemas com eficiência espectral 2 bits/s/Hz.

Os resultados obtidos estão representados na Figura 2, onde estão apresentados os desempenhos do sistema aqui proposto para duas MCW, $2 \times 8$ e $2 \times 128$ e um sistema de codificação wavelet que utiliza uma MCW $2 \times 128$, eficiência espectral de $1 \mathrm{bit} / \mathrm{s} / \mathrm{Hz}$ e uma constelação 11-PSK especialmente projetada [10].

Pode-se observar que o sistema de maior eficiência espectral aqui proposto mantém o bom desempenho dos sistemas wavelet, e mesmo com uma matriz $2 \times 8$ tem desempenho próximo ao sistema 11-PSK nos baixos valores de SNR. Ao utilizar a matriz $2 \times 128$ o desempenho do sistema melhora, e supera o do esquema 11-PSK em todos os valores de SNR.

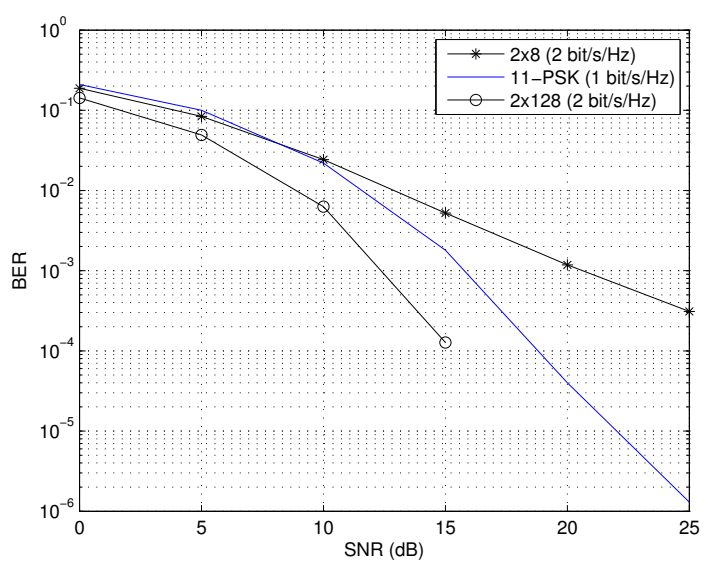

Fig. 2. Curvas de BER para sistemas wavelet 11-PSK (1 bit/s/Hz) e eficiência espetral 2 bits/s/Hz com MCW 2x8 e 2x128.

Ainda para efeito de avaliação do sistema obtido, na Figura 3 estão representados os desempenhos do sistema com eficiência espectral de $2 \mathrm{bits} / \mathrm{s} / \mathrm{Hz}$ aqui proposto, um para cada matriz wavelet, e o desempenho do sistema com codificação espácio-temporal também com 2 bits/s/Hz, porém com quatro antenas de transmissão e uma de recepção usando constelação 16-QAM [11] e código de taxa 1/2.

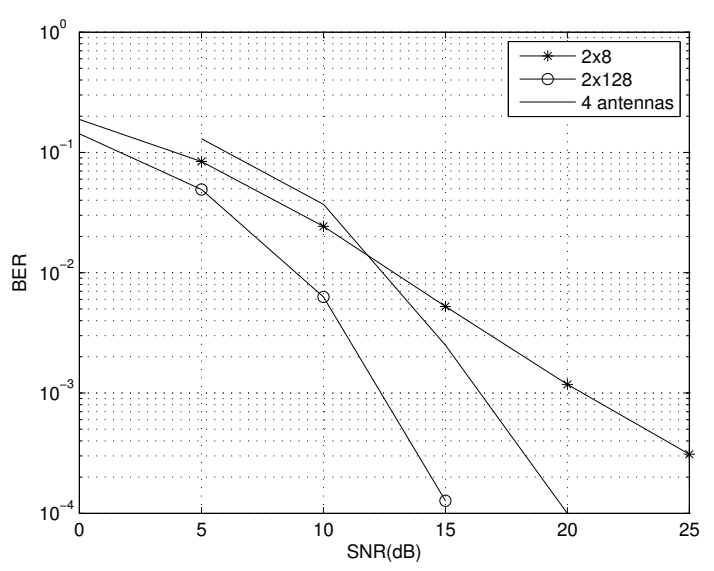

Fig. 3. Curvas de BER para sistemas wavelet com espetral 2 bits/s/Hz com MCW 2x8 e 2x128, e um sistema com codificação espácio-temporal de bloco com 4 antenas de transmissão, uma de recepção e sinais 16-QAM com código de taxa $1 / 2$.

Inicialmente deve-se observar que o sistema aqui proposto utiliza apenas uma antena de transmissão e uma de recepção, enquanto o sistema apresentado em [11] possui forte diversidade espacial, porém com quatro antenas de transmissão e uma na recepção. A diferença de $4 \mathrm{~dB}$ é significativa sugerindo o potencial dos esquemas de codificação com uso de matrizes wavelet ainda que se considere que a constelação é do tipo multinível.

\section{CONCLUSÕES}

Em trabalhos anteriores foi demonstrado o bom desempenho de sistemas com codificação wavelet, porém em todos os 
sistemas previamente apresentados a eficiência espectral era constante e igual a $1 \mathrm{bit} / \mathrm{s} / \mathrm{Hz}$. Neste trabalho foi apresentada uma técnica para combinar sequências de símbolos wavelet e com isso aumentar a eficiência espectral.

Utilizando a expansão de uma função geradora de momentos mostrou-se através de um exemplo, que é possível obter quais as sequências de símbolos possíveis e suas probabilidades conjuntas. Observou-se que o agrupamento de sequências de símbolos wavelet pode ser vista como uma constelação de sinais, e que essa consideração leva a obtenção de uma constelação com boas características.

O desempenho do sistema proposto, com maior eficiência espectral e realizando uma codificação conjunta fonte-canal foi obtido via simulação computacional, e comparado com sistemas wavelet que utilizam constelações de sinais otimizadas, mas com eficiência espectral $1 \mathrm{bit} / \mathrm{s} / \mathrm{Hz}$.

Os resultados apresentados mostram que o sistema proposto além de ter sua eficiência espectral aumentada, ainda consegue obter um desempenho melhor que sistemas wavelet com constelações especialmente projetadas.

O método proposto pode ser utilizado pra obter sistemas de codificação wavelet com eficiência espectral mais altas dos que a do exemplo aqui apresentado, para tanto será necessário projetar formas de combinar as sequências de símbolos, esse é um tema para trabalhos futuros. Além disso, tendo sistemas com maior eficiência espectral será possível implementar sistemas de codificação com taxa variável, por intermédio de uma simples alteração na "superposição" das linhas da matriz de codificação.

\section{REFERÊNCIAS}

[1] M.A. Tzannes and M.C Tzannes, Bit-by-bit channel coding using wavelets. Global Telecommunications Conference, 1992. Conference Record., GLOBECOM '92. Communication for Global Users., IEEE, 1992, pp. 684-688, vol.2.

[2] Resnikoff, H. L. W. Wavelet Analysis, The Scalable Structure of Information Springer, 1998.

[3] Silveira, L.F.Q., de Assis, F.M. and Pinto, E.L., A new wavelet coded $P S K$ system over time-varying flat fading channels. IEEE 5th Workshop on Signal Processing Advances in Wireless Communications (SPAWC 2004), pp. 429-423, July, 2004.

[4] Santos, E. A., Gurjão, E. C, de Assis, F. M.. Projeto de Constelações de Sinais para Codificação Wavelet. XXIX Simpósio Brasileiro de Telecomunicações (SBrT 2011), Outubro, 20011, Curitiba-PR, Brasil.

[5] Silveira, L.F.Q., Silveira, L.G.Q. and de Assis, F.M. and Pinto, E.L., Analysis and optimization of wavelet-coded communication systems. IEEE Transactions on Wireless Communications, pp. 563-567, vol. 8, no. 2, feb. 2009

[6] Pinto, E. L. e Albuquerque, C. P. de, A técnica de transmissão OFDM. Revista Científica Periódica, Vol.5, No 1, Junho de 2002.

[7] Silveira, L. F. Q., Silveira, L. G. Q., de Assis, F. M., Performance Analysis of Space Diversity Scheme In Wavelet-Coded Systems Over Fading Channels. International Microwave \& Optoelectronics Conference (IMOC 2009), pp. 277-281, Belém, PA, Brasil, 2009, DOI 10.1109/IMOC.2009.5427581

[8] Goulden, I. P., Jackson, D. M., Combinatorial Enumeration. WileyInterscience, 1983

[9] Yannis, V., Probability and random processes for electrical engineers. McGraw-Hill, 1998.

[10] Santos, E. A., Gurjão, E. C, de Assis, F. M.. Improved Optimization Algorithm for Constellation Mappings of Wavelet-Coded Communication Systems. International Workshop on Telecommunications, Santa Rita do Sapucaí, MG, Brasil, 2013.

[11] Tarokh, V., Jafakhani, H., Calderbank, A. R. Space-Time Block Coding for Wireless Communications: Performance Results. IEEE Transactions on Selected Areas in Communications, Vol. 17, No 3, March 1999. 